\title{
Étude des profils plasmatiques du cortisol, des acides aminés, du glucose et de l'urée après une injection d'ACTH chez la vache tarie
}

\author{
BB Ndibualonji, D Dehareng, JM Godeau
}

Université de Liège, faculté de Médecine vétérinaire, laboratoire de biochimie, avenue de Colonster, 20 (Bát B 42), 4000 Liège-Sart Tilman, Belgique

Ce travail a pour but de préciser chez les bovins l'évolution de l'aminoacidémie libre, de la glycémie et de l'urémie en réponse à une injection intraveineuse de l'hormone de stress (ACTH).

Deux vaches adultes taries et non gravides ont été alimentées en 2 repas quotidiens égaux (06 $\mathrm{h} 15-15 \mathrm{~h} 30$ ) constitués de foin et de concentré. Les vaches ont reçu à $9 \mathrm{~h}$ du matin $5 \mathrm{ml}$ de sérum physiologique (SER) par un cathéter placé dans la veine jugulaire. Deux semaines plus tard, à la même heure, elles ont reçu 6 UI d'ACTH (soit $240 \mu \mathrm{l}$ de synacthen dans $4,76 \mathrm{ml}$ de SER). Les échantillons de sang ont été prélevés à $8 \mathrm{~h}, 9 \mathrm{~h}$ $10,9 \mathrm{~h} \mathrm{20,9}$ h 30,10 h, $11 \mathrm{~h}, 12 \mathrm{~h}, 13 \mathrm{~h}, 14 \mathrm{~h}$, $15 \mathrm{~h}$. Le dosage du cortisol (Cor) a été fait par RIA, les acides aminés (AA : Asp, Gln, Val et Phe) par HPLC, le glucose (Glc) par une méthode enzymatique à l'hexokinase et l'urée (Ur) par une méthode colorimétrique adaptée au Technicon. Le test $t$ de Student a été utilisé pour analyser les différences moyennes entre les résultats des analyses obtenus après les 2 traitements. La cortisolémie basale $(2,8 \mathrm{ng} / \mathrm{ml})$ augmente de $1100 \%$ $(29,7 \mathrm{ng} / \mathrm{ml}) 10 \mathrm{~min}$ après injection d'ACTH, et atteint $58,2 \mathrm{ng} / \mathrm{ml}$ (maximum) après $1 \mathrm{~h}$. Le niveau basal est retrouvé $5 \mathrm{~h}$ plus tard. Une augmentation de $35 \%$ du cortisol a été obtenue $10 \mathrm{~min}$ après injection du SER mais les concentrations sont revenues à la normale après $30 \mathrm{~min}$. L'injection d'ACTH a induit une diminution ponctuelle maximale de $25 \%$ pour Asp et $26 \%$ pour GIn, et une augmentation ponctuelle maximale de $5 \%$ pour Val, $10 \%$ pour Phe et $15 \%$ pour Glc. L'uré- mie est restée inchangée après traitement. Par rapport au traitement témoin (SER), l'injection d'ACTH a montré sur toute la période de mesure (08-16 h) une diminution moyenne des concentrations pour l'Asp (56 \pm 7 vs $63 \pm 5 \mu \mathrm{M}$, $P<0,001)$ et la Gin $(305 \pm 28$ vs $333 \pm 19 \mu \mathrm{M}$, $P<0,01)$ et une augmentation moyenne significative pour Phe $(56 \pm 5$ vs $51 \pm 4 \mu \mathrm{M}, P<0,001)$ et non significative pour $\mathrm{Val}(202 \pm 13$ vs $193 \pm 11$ $\mu \mathrm{M})$ et Glc (74 \pm 8 vs $69 \pm 6 \mathrm{mg} / 100 \mathrm{ml}$ ). L'augmentation de la cortisolémie $10 \mathrm{~min}$ après injection de 6 UI d'ACTH de synthèse est en accord avec les résultats obtenus avec la même dose chez les vaches en lactation (Van der Kolk et al, 1991). De même, une augmentation de la glycémie $(50 \%)$ et des AA essentiels, une diminution des AA non essentiels et une absence de variation de l'urémie ont été observées après une infusion intraveineuse en continu de $0,25 \mathrm{UI} / \mathrm{h}$ de synacthen pendant $2 \mathrm{j}$ chez le mouton (Slater et Mellor, 1977).

En conclusion, l'hormone de stress (ACTH) a induit une libération immédiate du cortisol au niveau du cortex surrénalien et une diminution des acides aminés glucoformateurs, suggérant une utilisation de ceux-ci via la néoglucogenèse. Ceci expliquerait la tendance à l'augmentation de la glycémie observée chez les vaches après traitement à l'ACTH.

Slater JS, Mellor DJ (1977) Res Vet Sci22, 95-100

Van der Kolk JH, Breukink HJ, Wensing TH, Mol JA (1991) Vet Quart 13, 144-147 